\title{
Maximally Localized Wannier Orbitals and the Extended Hubbard Model for Twisted Bilayer Graphene
}

\author{
Mikito Koshino, ${ }^{1, *}$ Noah F. Q. Yuan, ${ }^{2}$ Takashi Koretsune, ${ }^{3}$ Masayuki Ochi, ${ }^{1}$ Kazuhiko Kuroki, ${ }^{1}$ and Liang Fu ${ }^{2}$ \\ ${ }^{1}$ Department of Physics, Osaka University, Toyonaka 560-0043, Japan \\ ${ }^{2}$ Department of Physics, Massachusetts Institute of Technology, Cambridge, Massachusetts 02139, USA \\ ${ }^{3}$ Department of Physics, Tohoku University, Sendai 980-8578, Japan
}

(Received 21 May 2018; published 28 September 2018)

\begin{abstract}
We develop an effective extended Hubbard model to describe the low-energy electronic properties of the twisted bilayer graphene. By using the Bloch states in the effective continuum model and with the aid of the maximally localized algorithm, we construct the Wannier orbitals and obtain an effective tight-binding model on the emergent honeycomb lattice. We find that the Wannier state takes a peculiar three-peak form in which the amplitude maxima are located at the triangle corners surrounding the center. We estimate the direct Coulomb interaction and the exchange interaction between the Wannier states. At the filling of two electrons per supercell, in particular, we find an unexpected coincidence in the direct Coulomb energy between a charge-ordered state and a homogeneous state, which could possibly lead to an unconventional many-body state.
\end{abstract}

DOI: 10.1103/PhysRevX.8.031087

Subject Areas: Condensed Matter Physics, Graphene, Strongly Correlated Materials

\section{INTRODUCTION}

The recent discovery of the superconductivity and strongly correlated insulating state in the twisted bilayer graphene (TBG) $[1,2]$ has attracted enormous attention and triggered a surge of theoretical works on this subject [3-16]. TBG is a bilayer system in which two graphene layers are rotationally stacked on each other [17-22], where the electronic band structure sensitively depends on the twist angle $\theta$. In a small $\theta$, in particular, a slight difference in the lattice orientation gives rise to a long-period moiré interference pattern, causing a substantial modification of the Dirac dispersion [23-34]. Theoretically, it was predicted that the Fermi velocity vanishes at certain $\theta$ 's called the magic angles, around which nearly flat bands with extremely narrow bandwidth emerge at low energy [28,33]. The superconductivity is actually observed around a magic angle of $1.05^{\circ}$, where the insulating phase and the superconducting phase occur around the filling of two electrons per supercell.

It is a challenging problem to theoretically describe the many-body physics in TBG. At $\theta=1.05^{\circ}$, the spatial period of the moire pattern is more than $10 \mathrm{~nm}$, and the number of carbon atoms in a unit cell exceeds 10000 . The

\footnotetext{
*koshino@phys.sci.osaka-u.ac.jp
}

Published by the American Physical Society under the terms of the Creative Commons Attribution 4.0 International license. Further distribution of this work must maintain attribution to the author(s) and the published article's title, journal citation, and DOI. electronic property of such a huge and complex system can be calculated efficiently by the effective continuum model, which captures the long-wavelength physics associated with the moire period [23,28,29,31,34-36]. However, the effective continuum energy spectrum still contains a number of energy bands in the low-energy region, and we need one more step to simplify the model so as to exclusively describe the nearly flat bands at lowest energy.

Actually, the nearly flat bands are separated by the energy gaps from other bands $[1,37,38]$, making it possible to construct an effective lattice model with well-localized Wannier orbitals purely consisting of the flat band states. Such an effective model was actually predicted by the symmetry analysis [4], which concludes that the Wannier orbitals should be centered at nonequivalent $\mathrm{AB}$ and $\mathrm{BA}$ spots in the moiré pattern, to form an emergent honeycomb lattice. Arguments and calculations suggesting a honeycomb lattice description have also been put forward in an independent work [5]. To obtain a concrete model with specific parameters, we need to construct the actual Wannier orbitals from the realistic model of TBG.

In this paper, we develop an extended Hubbard model of TBG at the magic angle $\left(\theta=1.05^{\circ}\right)$, based on the effective continuum model. By taking an appropriate linear combination of the Bloch states in the nearly flat bands, we build the Wannier orbitals centered at the AB and BA spots and obtain the effective tight-binding model on the emergent honeycomb lattice. Here, we adopt the maximally localized algorithm [39] to minimize the spread of the wave functions. From the obtained Wannier orbitals, we estimate the direct 
Coulomb energy and the exchange energy between electrons residing at different orbitals. The obtained Wannier state is centered at the $\mathrm{AB}$ or $\mathrm{BA}$ spot, while we find that its maximum amplitude is not at the center but at three AA spots surrounding the center, as also noticed in Ref. [5]. Importantly, the pair of Wannier orbitals that we constructed explicitly has $\left(p_{x}, p_{y}\right)$ on-site symmetry and hence forms a doublet under threefold rotation around their centers, consistent with the symmetry analysis [4].

Because of this peculiar three-peak form, the electronelectron interaction between the neighboring sites is as important as the on-site interaction term. At the filling of two electrons per supercell, in particular, we find an unexpected coincidence in the direct Coulomb energy between two different many-body states: a homogeneous state where an electron enters every sublattice of the effective honeycomb lattice and a charge-ordered state where two electrons reside at every two sublattices [Figs. 7(a) and 7(b)]. We expect that such competing nature would possibly give rise to a nontrivial many-body ground state.

This paper is organized as follows: In Sec. II, we explain the atomic structure of TBG, and in Sec. III, we introduce the effective continuum model and argue the structure of the nearly flat bands at the magic angle $\theta=1.05^{\circ}$. In Sec. IV, we construct the Wannier orbitals using the maximally localizing method, and we obtain the tightbinding model in Sec. V. We consider the electron-electron interaction between the Wannier states in Sec. VI. A brief conclusion is presented in Sec. VII.

\section{ATOMIC STRUCTURE}

We define the atomic structure of TBG by starting from AA-stacked bilayer graphene (i.e., perfectly overlapping honeycomb lattices) and rotating layers 1 and 2 around a pair of registered $B$ sites by $-\theta / 2$ and $+\theta / 2$, respectively. We define $\mathbf{a}_{1}=a(1,0)$ and $\mathbf{a}_{2}=a(1 / 2, \sqrt{3} / 2)$ as the lattice vectors of the initial AA-stacked bilayer before the rotation, where $a \approx 0.246 \mathrm{~nm}$ is the lattice constant of graphene. The corresponding reciprocal lattice vectors are $\mathbf{a}_{1}^{*}=(2 \pi / a)(1,-1 / \sqrt{3})$ and $\mathbf{a}_{2}^{*}=(2 \pi / a)(0,2 / \sqrt{3})$. After the rotation, the lattice vectors of layer $l$ are given by $\mathbf{a}_{i}^{(l)}=R(\mp \theta / 2) \mathbf{a}_{i}$, with $\mp$ for $l=1,2$, respectively, where $R(\theta)$ represents the rotation by $\theta$. Likewise, the reciprocal lattice vectors become $\mathbf{a}_{i}^{*(l)}=R(\mp \theta / 2) \mathbf{a}_{i}^{*}$. With respect to the registered $B$ sites, TBG has point group $D_{3}$ generated by a threefold in-plane rotation $C_{3 z}$ along the $z$ axis and a twofold rotation $C_{2 y}$ along the $y$ axis.

In a small angle TBG, the slight mismatch of the lattice periods of two layers gives rise to a long-period moiré interference pattern. The reciprocal lattice vectors for the moiré pattern are given by $\mathbf{G}_{i}^{\mathrm{M}}=\mathbf{a}_{i}^{*(1)}-\mathbf{a}_{i}^{*(2)}(i=1,2)$. The real-space lattice vectors $\mathbf{L}_{j}^{\mathrm{M}}$ can then be obtained from $\mathbf{G}_{i}^{\mathrm{M}} \cdot \mathbf{L}_{j}^{\mathrm{M}}=2 \pi \boldsymbol{\delta}_{i j}$. A moiré unit cell is spanned by $\mathbf{L}_{1}^{\mathrm{M}}$ and $\mathbf{L}_{2}^{\mathrm{M}}$. The lattice constant $L_{\mathrm{M}}=\left|\mathbf{L}_{1}^{\mathrm{M}}\right|=\left|\mathbf{L}_{2}^{\mathrm{M}}\right|$ is $L_{\mathrm{M}}=a /[2 \sin (\theta / 2)]$. Figure 1(a) illustrates the atomic structure of TBG with $\theta=3.89^{\circ}$. The lattice structure locally resembles the regular stacking such as $\mathrm{AA}, \mathrm{AB}$, or $\mathrm{BA}$, depending on the position, where AA represents the perfect overlapping of hexagons, and $\mathrm{AB}$ (BA) is the shifted configuration in which the $A_{1}\left(B_{1}\right)$ sublattice is right above $\mathrm{B}_{2}\left(\mathrm{~A}_{2}\right)$. In Fig. 1(a), AA spots are located at the crossing points of the grid lines, and $\mathrm{AB}$ and $\mathrm{BA}$ spots are at the centers of triangles indicated by dots. Figure 1(b)
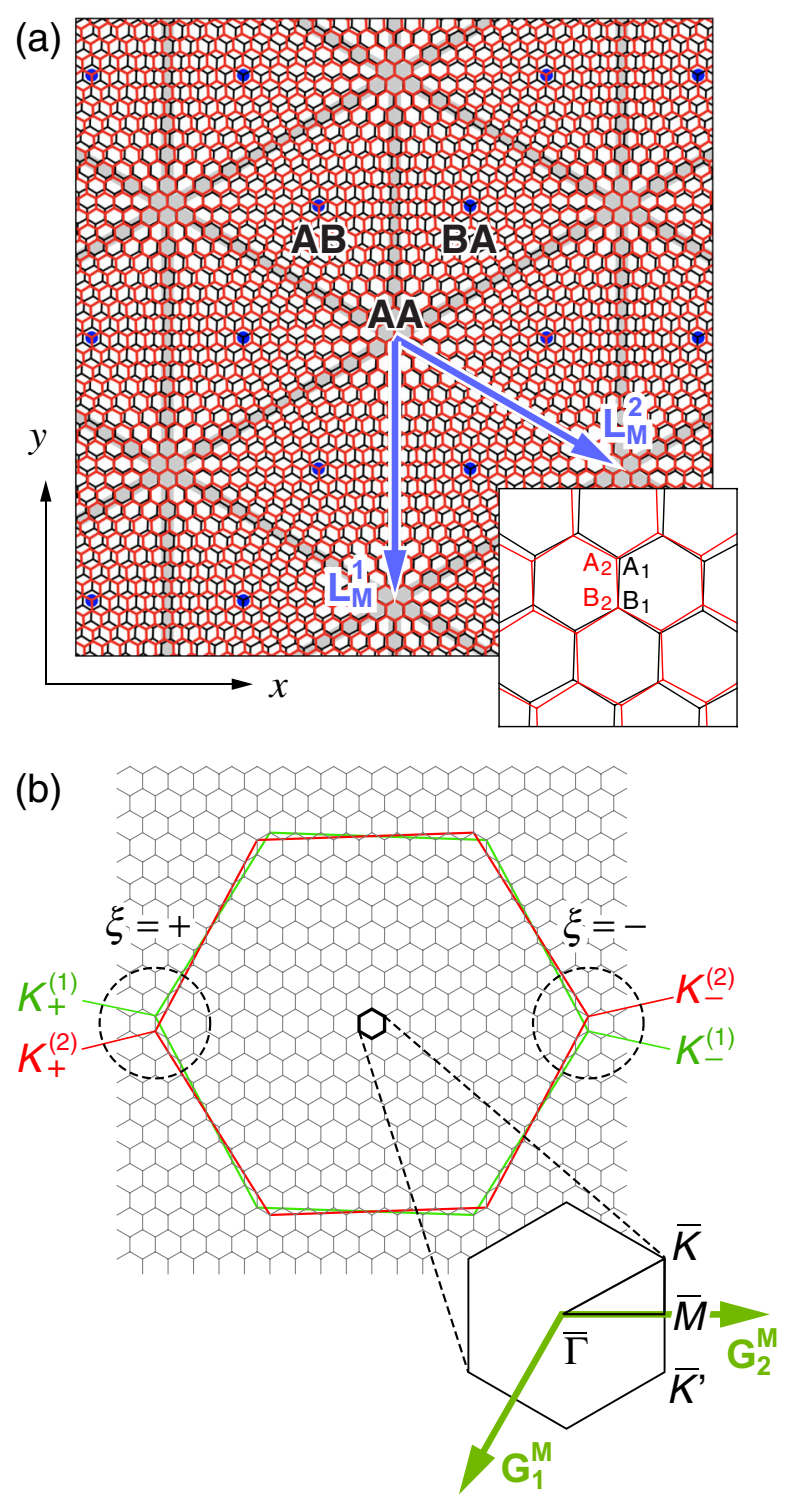

FIG. 1. (a) Atomic structure of TBG with $\theta=3.89^{\circ}$ and $D_{3}$ symmetry. AA spots are located at the crossing points of the grid lines, and $\mathrm{AB}$ and $\mathrm{BA}$ spots are at the centers of triangles indicated by dots. (b) Brillouin zone folding in TBG with $\theta=3.89^{\circ}$. Two large hexagons represent the first Brillouin zones of graphene layers 1 and 2, and the small hexagon is the moiré Brillouin zone of TBG. 
shows the corresponding folding of the Brillouin zone, where two large hexagons represent the first Brillouin zones of layers 1 and 2, and the small hexagon is the moiré Brillouin zone of TBG. The graphene's Dirac points (the band touching points) are located at $\mathbf{K}_{\xi}^{(l)}=-\xi\left[2 \mathbf{a}_{1}^{(l) *}+\right.$ $\left.\mathbf{a}_{2}^{(l) *}\right] / 3$ for layer $l$, where $\xi= \pm 1$ is the valley index. We label the symmetric points of the reduced Brillouin zone as $\bar{\Gamma}, \bar{M}, \bar{K}$, and $\bar{K}^{\prime}$ as in Fig. 1(b).

We can construct the TBG in alternative manners, for example, by rotating around the hexagon centers instead of the B site. In that case, we have the different superlattice structure with point group $D_{6}$. For completeness, we leave the discussion of the $D_{6}$ structure and other superlattice structures to the Supplemental Material [40].

\section{EFFECTIVE CONTINUUM MODEL}

When the moiré period is much longer than the atomic scale, the electronic structure can be described by an effective continuum model [23,28,29,31,34-36]. Here, the intervalley mixing between $\xi= \pm$ can be safely neglected, and the total Hamiltonian is block-diagonalized into the two independent valleys. The effective Hamiltonian of the continuum model for the valley $\xi$ is written in a $4 \times 4$ matrix for the basis of $\left(A_{1}, B_{1}, A_{2}, B_{2}\right)$ as

$$
H^{(\xi)}=\left(\begin{array}{cc}
H_{1} & U^{\dagger} \\
U & H_{2}
\end{array}\right) \text {. }
$$

Here, $H_{l}(l=1,2)$ is the intralayer Hamiltonian of layer $l$, which is given by the two-dimensional Weyl equation centered at the $\mathbf{K}_{\xi}^{(l)}$ point,

$$
H_{l}=-\hbar v\left[R( \pm \theta / 2)\left(\mathbf{k}-\mathbf{K}_{\xi}^{(l)}\right)\right] \cdot\left(\xi \sigma_{x}, \sigma_{y}\right),
$$

where \pm is for $l=1$ and 2, respectively. We take $\hbar v / a=$ $2.1354 \mathrm{eV}$ [34]. Note that $U$ is the effective interlayer coupling given by [34-36]

$$
\begin{aligned}
U= & \left(\begin{array}{ll}
U_{A_{2} A_{1}} & U_{A_{2} B_{1}} \\
U_{B_{2} A_{1}} & U_{B_{2} B_{1}}
\end{array}\right) \\
= & \left(\begin{array}{cc}
u & u^{\prime} \\
u^{\prime} & u
\end{array}\right)+\left(\begin{array}{cc}
u & u^{\prime} \omega^{-\xi} \\
u^{\prime} \omega^{\xi} & u
\end{array}\right) e^{i \xi \mathbf{G}_{1}^{\mathrm{M}} \cdot \mathbf{r}} \\
& +\left(\begin{array}{cc}
u & u^{\prime} \omega^{\xi} \\
u^{\prime} \omega^{-\xi} & u
\end{array}\right) e^{i \xi\left(\mathbf{G}_{1}^{\mathrm{M}}+\mathbf{G}_{2}^{\mathrm{M}}\right) \cdot \mathbf{r}}
\end{aligned}
$$

where $\omega=e^{2 \pi i / 3}$. Here, $u$ and $u^{\prime}$ describe the amplitudes of diagonal and off-diagonal terms, respectively, in the sublattice space. The effective models in the previous studies [34-36] assume $u=u^{\prime}$, which corresponds to a flat TBG in which the interlayer spacing $d$ is constant everywhere. On the other hand, several theoretical studies predicted that the optimized lattice structure of TBG is actually corrugated in the out-of-plane direction, in such a way that $d$ is the widest in the $\mathrm{AA}$ stacking region and the narrowest in the $\mathrm{AB} / \mathrm{BA}$ stacking region [41-44]. Here, we incorporate the corrugation effect as a difference between $u=0.0797 \mathrm{eV}$ and $u^{\prime}=0.0975 \mathrm{eV}$ in the effective model, a detailed derivation of which is presented in the Appendix. As we show in the following, the difference between $u$ and $u^{\prime}$ introduces energy gaps between the lowest bands and the excited bands, in qualitative agreement with the experimental observation $[1,2,37]$. It was found that the energy gaps isolating the lowest nearly flat bands are also caused by the in-plane distortion [38].

We perform the calculation of the energy bands and the eigenstates in the $k$-space picture. For a single Bloch vector $\mathbf{k}$ in the moiré Brillouin zone, the moiré interlayer coupling hybridizes the graphene's eigenstates at $\mathbf{q}=$ $\mathbf{k}+\mathbf{G}$, where $\mathbf{G}=m_{1} \mathbf{G}_{1}^{\mathrm{M}}+m_{2} \mathbf{G}_{2}^{\mathrm{M}}$ and $m_{1}$ and $m_{2}$ are integers. Therefore, the eigenstate is written as

$$
\psi_{n \mathbf{k}}^{X}(\mathbf{r})=\sum_{\mathbf{G}} C_{n \mathbf{k}}^{X}(\mathbf{G}) e^{i(\mathbf{k}+\mathbf{G}) \cdot \mathbf{r}},
$$

where $X=A_{1}, B_{1}, A_{2}, B_{2}$ is the sublattice index, $n$ is the band index, and $\mathbf{k}$ is the Bloch wave vector in the moiré Brillouin zone. As the low-energy states are expected to be dominated by the individual graphene eigenstates near the original Dirac points, we pick up q's inside the cutoff circle $\left|\mathbf{q}-\mathbf{q}_{0}\right|<q_{c}$, where $\mathbf{q}_{0}$ is taken as the midpoint between $\mathbf{K}_{\xi}^{(1)}$ and $\mathbf{K}_{\xi}^{(2)}$, and $q_{c}$ is set to $4 G_{\mathrm{M}}\left(G_{\mathrm{M}}=\left|\mathbf{G}_{1}^{\mathrm{M}}\right|=\left|\mathbf{G}_{2}^{\mathrm{M}}\right|\right)$. Since the intervalley coupling can be neglected, the calculation is performed independently for each of $\xi= \pm$ as we discussed previously. We then numerically diagonalize the Hamiltonian within the limited wave space inside the cutoff circle and obtain the eigenenergies and eigenstates.

Figure 2(a) shows the energy band and the density of states of TBG at the magic angle $\theta=1.05^{\circ}$, calculated using the approach mentioned. Here and in the following, the origin of the band energy axis is set to the charge neutral point. The lower panel is the enlarged plot of the zeroenergy region where the nearly flat bands are located. The black solid line and red dashed line represent the energy bands of $\xi= \pm$ valleys, respectively. They are the timereversal partners of each other, and the energy bands of $\xi=-$ are obtained by inverting $\mathbf{k}$ to $-\mathbf{k}$. The flat band cluster consists of two bands per spin and valley, which are denoted as $E_{1}(\mathbf{k})$ and $E_{2}(\mathbf{k})$ for the hole side and the electron side, respectively. The overall structure is about $7.5 \mathrm{meV}$ wide in the energy axis and separated from the excited bands by an energy gap of about $14 \mathrm{meV}$ in both the electron side and the hole side. Figure 2(b) shows the contour plots of $E_{1}(\mathbf{k})$ and $E_{2}(\mathbf{k})$ for the valley $\xi=+$. Note that $E_{1}(\mathbf{k})$ and $E_{2}(\mathbf{k})$ are trigonally warped in opposite directions, so $E_{1}(\mathbf{k}) \neq E_{1}(-\mathbf{k})$ and $E_{2}(\mathbf{k}) \neq E_{2}(-\mathbf{k})$. The particle-hole symmetry is absent, and the $E_{1}$ band is wider than the $E_{2}$ band. The van Hove singularity is located at $E \approx-0.11 \mathrm{meV}$ and $0.16 \mathrm{meV}$, which correspond to the carrier density $n / n_{0} \approx-0.78$ and 0.63 , respectively, with 

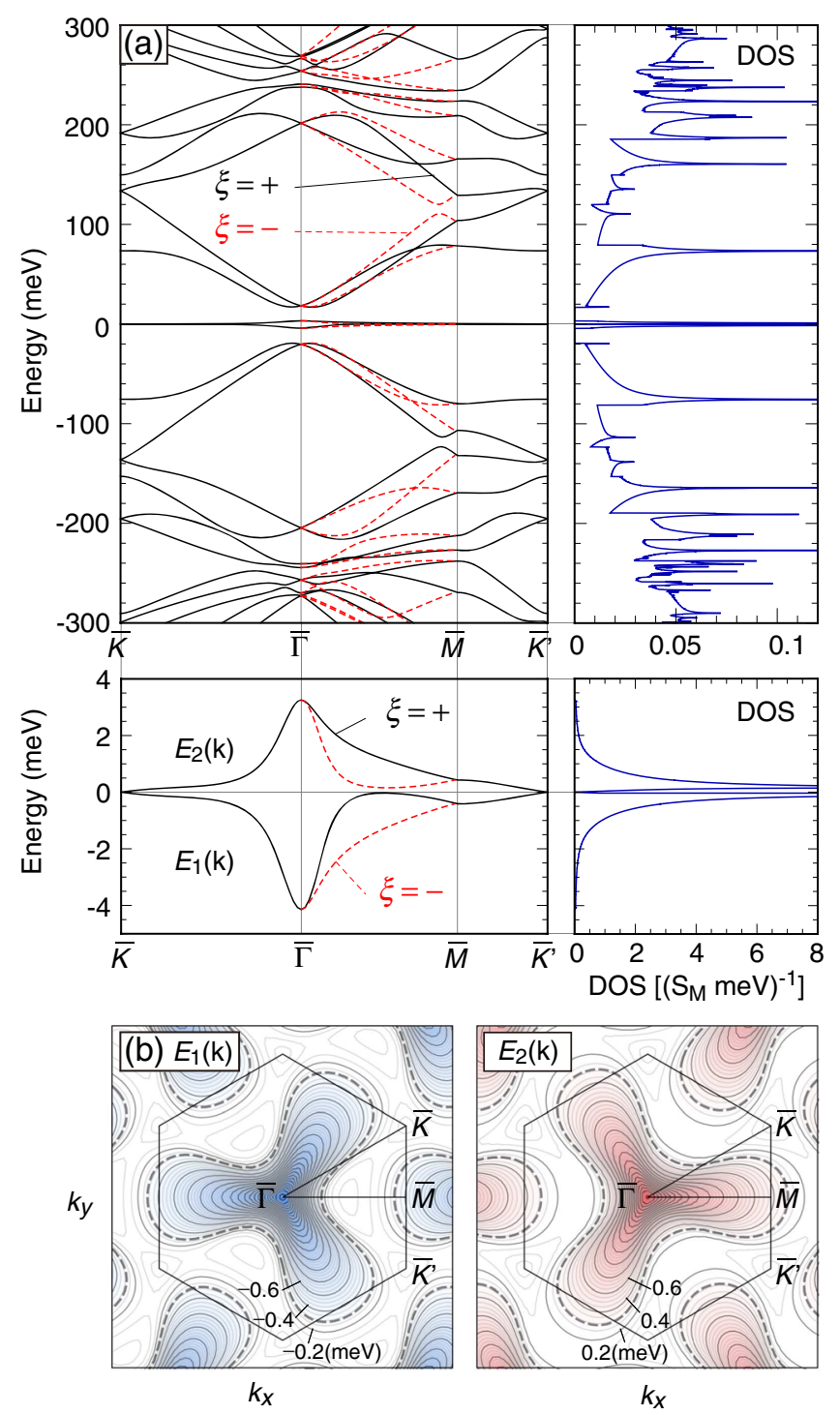

FIG. 2. (a) Energy band and the density of states of TBG at $\theta=1.05^{\circ}$, where the lower panel is the enlarged plot of the zero-energy region. The black solid line and red dashed line represent the energy bands of $\xi= \pm$ valleys, respectively. (b) Contour plots of $E_{1}(\mathbf{k})$ and $E_{2}(\mathbf{k})$ for the valley $\xi=+$. The dashed contour corresponds to the filling of two electrons/ holes per supercell $\left(n / n_{0}= \pm 2\right)$.

spin and valley included. Here, $n_{0}=1 / S_{\mathrm{M}}, S_{\mathrm{M}}=$ $(\sqrt{3} / 2) L_{M}^{2}$ is the moire unit area (the band gap is $\left.n / n_{0}= \pm 4\right)$ and $L_{\mathrm{M}}$ is $13.4 \mathrm{~nm}$ at $\theta=1.05^{\circ}$. The filling of two electrons/holes per supercell $\left(n / n_{0}= \pm 2\right)$ corresponds to $E \approx 0.289 \mathrm{meV}$ and $-0.286 \mathrm{meV}$, respectively, which are indicated by dashed contours in Fig. 2(b).

\section{WANNIER ORBITALS}

We construct the localized Wannier orbitals from the Bloch wave functions of the effective model. Since the nearly flat bands are energetically isolated from other bands, we expect that well-localized orbits can be made purely from the flat band states, with all other bands neglected. The number of independent Wannier orbitals in a unit cell coincides with the number of energy bands taken into account, so we have two Wannier orbitals per spin and valley. According to the symmetry analysis [4], the two orbitals should be centered at the $\mathrm{AB}$ and $\mathrm{BA}$ spots to form a honeycomb lattice. Our strategy is to first prepare certain initial orbitals centered at $\mathrm{AB}$ and $\mathrm{BA}$ and then apply the maximally localized algorithm [39]. The following process is applied to $\xi= \pm$ valleys separately, and we omit the valley index $\xi$ hereafter.

The initial wave functions can be prepared as follows. First, we fix the global phase factor of the Bloch states in two different ways: In gauge 1, we fix the phase so that $\psi_{n \mathbf{k}}^{B_{1}}\left(\mathbf{r}_{\mathrm{BA}}\right)$ is real, and in gauge 2 , we fix the phase so that $\psi_{n \mathbf{k}}^{A_{1}}\left(\mathbf{r}_{\mathrm{AB}}\right)$ is real. Here, $\mathbf{r}_{\mathrm{BA}}=(1 / 2, \sqrt{3} / 2)\left(L_{\mathrm{M}} / \sqrt{3}\right)$ and $\mathbf{r}_{\mathrm{AB}}=(-1 / 2, \sqrt{3} / 2)\left(L_{\mathrm{M}} / \sqrt{3}\right)$ are the positions of the BA and $\mathrm{AB}$ spots, respectively, measured from the AA spot $(0,0)$ [Fig. 1(a)]. We write the Bloch function in gauge 1 as $\psi_{n \mathbf{k}}$ and that in gauge 2 as $e^{i \phi_{n \mathbf{k}}} \psi_{n \mathbf{k}}$, where $e^{i \phi_{n \mathbf{k}}}$ is the relative phase factor between gauges 1 and 2 . We construct the initial Wannier orbitals 1 and 2 by summing the Bloch states of the bands $\psi_{1 \mathbf{k}}$ and $\psi_{2 \mathbf{k}}$ [corresponding to $E_{1}(\mathbf{k})$ and $E_{2}(\mathbf{k})$, respectively] as

$$
\begin{aligned}
|\mathbf{R}, 1\rangle_{0} & =\frac{1}{\sqrt{N}} \sum_{\mathbf{k}} e^{-i \mathbf{k} \cdot \mathbf{R}} \frac{1}{\sqrt{2}}\left(\left|\psi_{1 \mathbf{k}}\right\rangle+\left|\psi_{2 \mathbf{k}}\right\rangle\right), \\
|\mathbf{R}, 2\rangle_{0} & =\frac{1}{\sqrt{N}} \sum_{\mathbf{k}} e^{-i \mathbf{k} \cdot \mathbf{R}} \frac{1}{\sqrt{2}} e^{i \phi_{1 \mathbf{k}}}\left(\left|\psi_{1 \mathbf{k}}\right\rangle-\left|\psi_{2 \mathbf{k}}\right\rangle\right) .
\end{aligned}
$$

Here, $\mathbf{R}=n_{1} \mathbf{L}_{1}^{\mathrm{M}}+n_{2} \mathbf{L}_{2}^{\mathrm{M}}$ is the moire lattice vector, and the summation in $\mathbf{k}$ is taken over $N$ discrete points in the moire Brillouin zone. We take $N=18 \times 18$ in this study. It is straightforward to check the orthonormality, ${ }_{0}\left\langle\mathbf{R}^{\prime}, n^{\prime} \mid \mathbf{R}, n\right\rangle_{0}=\boldsymbol{\delta}_{\mathbf{R}, \mathbf{R}^{\prime}} \boldsymbol{\delta}_{n, n^{\prime}}$.

While $|\mathbf{R}, 1\rangle_{0}$ and $|\mathbf{R}, 2\rangle_{0}$ are already well localized around the center positions $\mathbf{R}+\mathbf{r}_{\mathrm{BA}}$ and $\mathbf{R}+\mathbf{r}_{\mathrm{AB}}$, respectively, we can even reduce the spread of the wave function using a maximally localizing method [39]. The final expression for the orbital $n(=1,2)$ is given by

$$
|\mathbf{R}, n\rangle=\frac{1}{\sqrt{N}} \sum_{\mathbf{k}} e^{-i \mathbf{k} \cdot \mathbf{R}} \sum_{m=1,2} U_{m n}^{(\mathbf{k})}\left|\psi_{m \mathbf{k}}\right\rangle,
$$

where $U_{m n}^{(\mathbf{k})}$ is a $2 \times 2$ unitary matrix. The algorithm optimizes $U_{m n}^{(\mathbf{k})}$ to minimize the spread functional. We set Eq. (5) as the initial value of $U_{m n}^{(\mathbf{k})}$ and iterate the minimization process until the convergence. In each step, we impose the symmetry constraint on $U_{m n}^{(k)}$. The optimized Wannier orbitals for the valley $\xi=+$ are illustrated in Fig. 3. Those for the opposite valley $\xi=-$ are given by the complex conjugate. For both orbitals 1 and 2, the top five 


\section{Orbital 1}
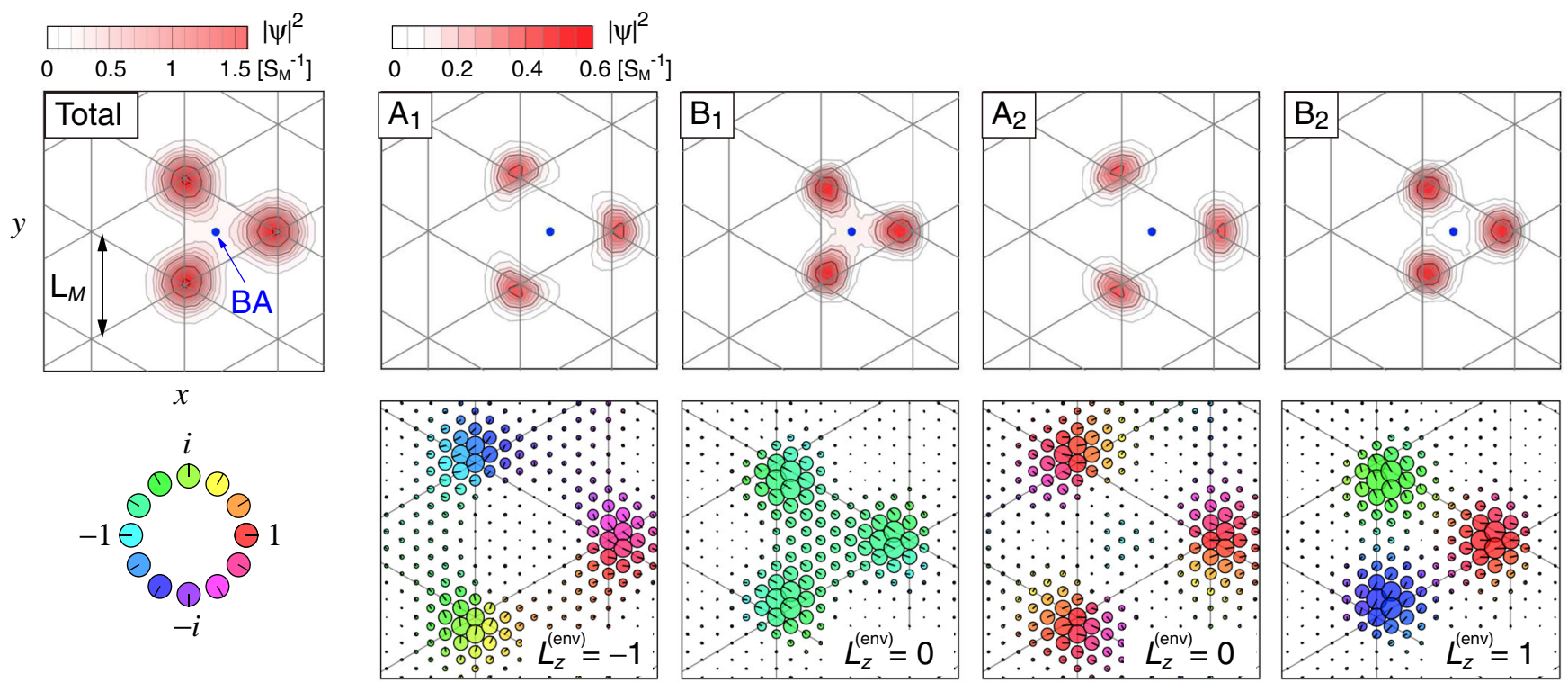

Orbital 2
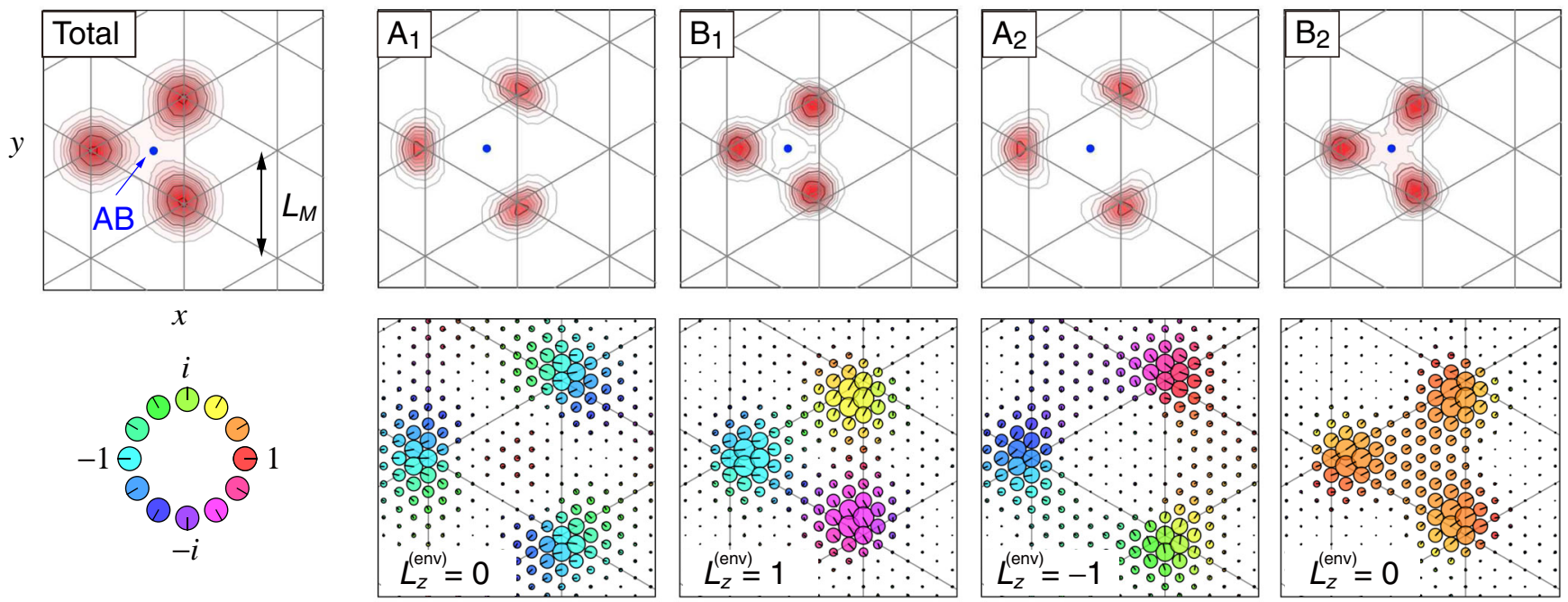

FIG. 3. Maximally localized Wannier orbitals of the valley $\xi=+$, in the low-energy flat band of TBG with $\theta=1.05^{\circ}$. For both orbitals 1 and 2, the top five panels show the contour maps for the squared amplitudes of the total wave function and of the four sublattice components. The lower panels illustrate the phase of the envelope function on some sample points, where the amplitude is indicated by the radius of a circle, and its phase factor is shown by the direction of the bar and also by color.

panels show the contour maps for the squared amplitudes of the total wave function and of the four sublattice components. We actually see that orbitals 1 and 2 are centered at the $\mathrm{BA}$ and $\mathrm{AB}$ positions, respectively, while the maxima of the wave amplitudes are located not at the center but near three AA spots surrounding the center. This reflects the fact that the Bloch wave functions of the nearly flat bands are mostly localized AA spots of the moiré pattern [25,41].

The lower panels illustrate the phase of the envelope function $F^{X_{l}}(\mathbf{r})(X=A, B$ and $l=1,2)$ on some sample points, where the total wave function is $\psi^{X_{l}}(\mathbf{r})=$ $e^{i \mathbf{K}_{\xi}^{(l)} \cdot \mathbf{r}} F^{X_{l}}(\mathbf{r})$. Here, the absolute value of $F^{X_{l}}(\mathbf{r})$ is indicated by the radius of a circle, and its phase factor is shown by the direction of the bar and also by color. Now, we see that the envelope functions on different sublattices have different eigenvalues of $C_{3 z}^{\prime}$, in-plane rotation with respect to its own center. However, noting that the Bloch factor $e^{i \mathbf{K}_{\xi} \cdot \mathbf{r}}$ also carries a nonzero eigenvalue of $C_{3 z}^{\prime}$, the total wave function $\psi=\left(\psi^{A_{1}}, \psi^{B_{1}}, \psi^{A_{2}}, \psi^{B_{2}}\right)$ is found to be an eigenstate of $C_{3 z}^{\prime}$ with a single eigenvalue. In orbital 1 , for example, the $C_{3 z}^{\prime}$ eigenvalue of $F^{X_{l}}$ is $\left(\omega, 1,1, \omega^{*}\right)$ for $\left(A_{1}, B_{1}, A_{2}, B_{2}\right)$, so the angular momentum of the envelope function is written as $L_{z}^{(\text {env })}=(-1,0,0,1)$. On the other hand, the $C_{3 z}^{\prime}$ eigenvalue for the Bloch factor $e^{i \mathbf{K}_{\xi} \cdot \mathbf{r}}$ can be found by noting that the BA 
spot (the orbital center) coincides with the $A_{1}$ site and the center of the hexagon of layer 2 [Fig. 1(a)], and then, we obtain $L_{z}^{\text {(Bloch) }}=(0,-1,-1,1)$. Therefore, the total angular momentum $L_{z}=L_{z}^{\text {(env) }}+L_{z}^{\text {(Bloch) }}$ is -1 for all the sublattices. Similarly, we can also show $L_{z}=-1$ for orbital 2 . Since the Wannier functions at the opposite valleys are related by the complex conjugate, we finally conclude that the eigenvalue of $C_{3 z}^{\prime}$ is $\omega^{\xi}=e^{\xi 2 \pi i / 3}$ for both orbitals 1 and 2. Namely, orbitals 1 and 2 from the same valley $\xi$ have the same nonzero angular momentum $L_{z}=-\xi$, in accordance with the symmetry analysis [4].

The initial guess of the Wannier orbital in Eq. (5) is closely related to the angular momentum of the envelope function. For orbital 1, the envelope function of $B_{1}$ has zero angular momentum, so it has a finite amplitude at the orbital center $\mathbf{r}_{\mathrm{BA}}$ as seen in Fig. 3. It does not contradict with the nonzero total angular momentum $L_{z}=-1$ because the BA spot does not coincide with the $B_{1}$ site. The finite amplitude at $\mathbf{r}_{\mathrm{BA}}$ is actually linked to the gauge choice for $|\mathbf{R}, 1\rangle_{0}$, which requires that $\psi_{n \mathbf{k}}^{B_{1}}\left(\mathbf{r}_{\mathrm{BA}}\right)$ is real. In the summation in $\mathbf{k}$, all the wave functions add up in the same phase at $r_{\mathrm{BA}}$, so we have an orbital localized at $\mathbf{r}_{\mathrm{BA}}$ with finite amplitude. The same is true for orbital 2, in which the envelope angular momentum vanishes at $A_{1}$. The wrong gauge choices [e.g., $\psi_{n \mathbf{k}}^{A_{1}}\left(\mathbf{r}_{\mathrm{BA}}\right)$ is real] do not make a well-localized orbital because the angular momentum of the Wannier function is forced by symmetry. Also, the hybridized form of $\left|\psi_{1 \mathbf{k}}\right\rangle \pm\left|\psi_{2 \mathbf{k}}\right\rangle$ in Eq. (5) localizes the wave function better than just using $\left|\psi_{1 \mathbf{k}}\right\rangle,\left|\psi_{2 \mathbf{k}}\right\rangle$. This is similar to monolayer graphene having the same honeycomb lattice structure, where the superposition of the positive and negative energy states is required to have an $A$-site or a $B$-site localized orbital.

\section{EFFECTIVE TIGHT-BINDING MODEL}

From the Wannier orbitals and the energy bands, we can derive the effective tight-binding model to exactly reproduce the dispersion of the nearly flat bands. In a straightforward calculation, the hopping integral between the Wannier orbitals is written as

$$
\begin{aligned}
& \left\langle\mathbf{R}^{\prime}, n^{\prime}|H| \mathbf{R}, n\right\rangle \\
& =\frac{1}{N} \sum_{\mathbf{k}} e^{i \mathbf{k} \cdot\left(\mathbf{R}^{\prime}-\mathbf{R}\right)}\left[\hat{U}^{(\mathbf{k}) \dagger}\left(\begin{array}{cc}
E_{1}(\mathbf{k}) & 0 \\
0 & E_{2}(\mathbf{k})
\end{array}\right) \hat{U}^{(\mathbf{k})}\right]_{n^{\prime} n},
\end{aligned}
$$

where $\hat{U}^{(\mathbf{k})}$ represents the matrix $U_{m n}^{(\mathbf{k})}$, and we use $\left\langle\psi_{n^{\prime} \mathbf{k}^{\prime}}|H| \psi_{n \mathbf{k}}\right\rangle=\boldsymbol{\delta}_{n n^{\prime}} \boldsymbol{\delta}_{\mathbf{k k}^{\prime}} E_{n}(\mathbf{k})$. In Figs. 4(a) and 4(b), we plot the hopping integrals from orbitals 1 and 2, respectively, for the valley $\xi=+$. Here, the honeycomb lattice represents the network of BA spots (orbital 1) and $\mathrm{AB}$ spots (orbital 2). The radius of the circle at each lattice point indicates the absolute value of the hopping integral from the origin (green circle at the center) to that point, and

\section{(a) From orbital 1}

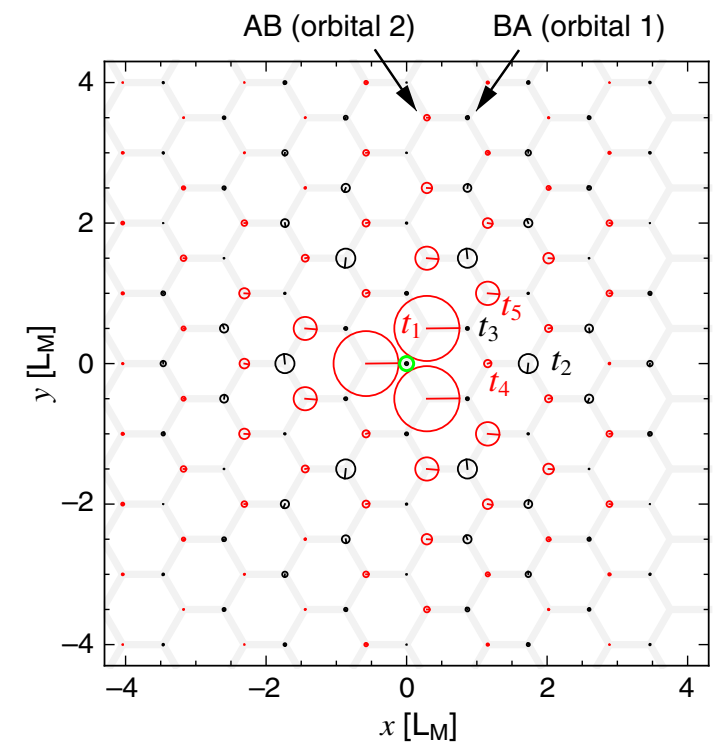

(b) From orbital 2

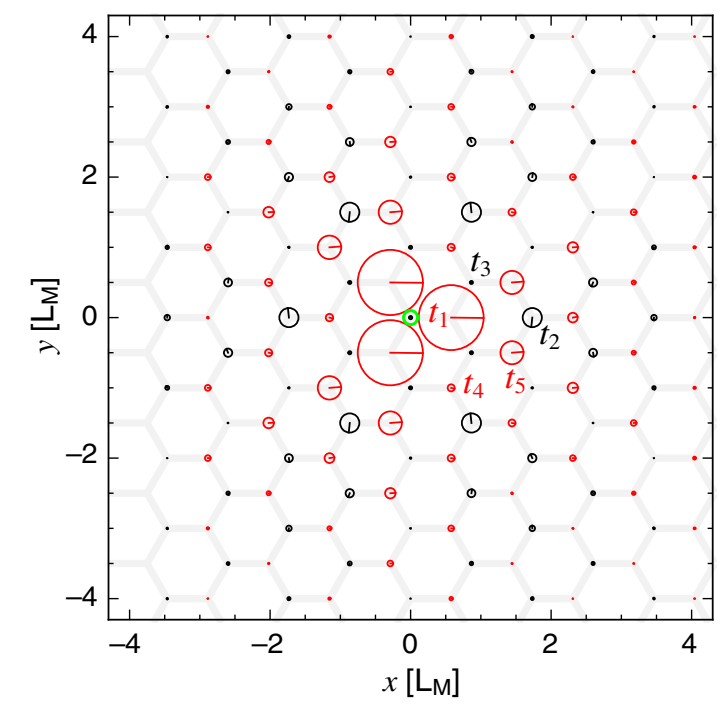

FIG. 4. Hopping integrals in the effective tight-binding model for the low-energy flat band of TBG at $\theta=1.05^{\circ}$. Panels (a) and (b) present the hopping parameters from the Wannier orbitals 1 and 2, respectively, where the radius of the circle at each lattice point indicates the absolute value of the hopping integral from the origin to that point, and the direction of the bar represents the phase in the complex plane.

the direction of the bar represents the phase in the complex plane. The effective tight-binding model for the valley $\xi=-$ is given by taking the complex conjugate. The list of the hopping integrals for the valley $\xi=+$ is included in the Supplemental Material [45].

To understand this effective tight-binding model, we need to analyze the symmetry properties of Wannier orbitals under point group $D_{3}$, as in Ref. [4]. Recall that orbitals 1 and 2 have nonzero angular momentum $L_{z}=-\xi$ at the valley $\xi$. Furthermore, under twofold rotation $C_{2 y}$, 
which interchanges two graphene layers, we find that orbital 1 from valley $\xi$ is mapped to orbital 2 from valley $-\xi$ and vice versa, as shown in Fig. 3. Hence, we can regard orbitals 1 and 2 from valley $\xi$ as the $p$-wave-like orbitals $p_{\xi} \equiv p_{x}+i \xi p_{y}$ residing on $\mathrm{BA}$ and $\mathrm{AB}$ spots, respectively. The angular momentum of the $p_{\xi}$ orbital is $L_{z}=-\xi$, whether its center is at a BA or AB spot, which is consistent with $L_{z}$ of orbitals 1 and 2. Under $C_{2 y}$, the two graphene layers, and hence $\mathrm{BA}$ and $\mathrm{AB}$ spots, are interchanged, and $\left(p_{x}, p_{y}\right) \rightarrow\left(-p_{x}, p_{y}\right)$ or $p_{\xi} \rightarrow-p_{-\xi}$. In other words, $C_{2 y}$ interchanges the $p_{\xi}$ orbital at the $\mathrm{BA}$ spot and the $p_{-\xi}$ orbital at the $\mathrm{AB}$ spot, which reproduces the symmetry transformation of orbitals 1 and 2 under $C_{2 y}$.

Once we identify the symmetries of orbitals 1 and 2 , the tight-binding model then describes hopping among $\left(p_{x}, p_{y}\right)$ orbitals on the honeycomb lattice formed by the $\mathrm{BA}$ and $\mathrm{AB}$ spots, which reads

$$
H=\sum_{\xi= \pm} \sum_{i j} t\left(\boldsymbol{r}_{i j}\right) e^{i \xi \phi\left(\boldsymbol{r}_{i j}\right)} c_{i \xi}^{\dagger} c_{j \xi}
$$

where $c_{i \xi}$ annihilates a $p_{\xi}$-orbital electron at site $i, \boldsymbol{r}_{i j}$ is the vector from site $i$ to $j$, and $t(\boldsymbol{r}), \phi(\boldsymbol{r})$ are as shown in Figs. 4(a) and 4(b).

The symmetry group of the tight-binding model of Eq. (8) is $G=D_{3} \times U(1) \times \mathrm{SU}(2) \times T$, where $D_{3}$ is the point group of TBG, which acts jointly on lattice sites and $\left(p_{x}, p_{y}\right)$ orbitals, $\mathrm{U}(1)$ acts in orbital space, $\mathrm{SU}(2)$ acts in spin space, and $T$ is the time-reversal symmetry. As discussed in Ref. [4], the microscopic origin of this orbital $\mathrm{U}(1)$ symmetry is that at small twist angles, the intervalley coupling is strongly suppressed, leading to this approximate valley conservation that exists independent of crystal symmetries.

The hopping integral $t(\boldsymbol{r})$ roughly decays with increasing $r=|\boldsymbol{r}|$. To include dominant contributions, we consider the nearest five hopping integrals $t_{1}$ to $t_{5}$ shown in Figs. 4(a) and 4(b), which are within the range $r \leq \sqrt{3} L_{\mathrm{M}}$. Notice that the subscripts are not labeled according to $r$. In the present model, we have $t_{1} \approx 0.331 \mathrm{meV}, t_{2} \approx(-0.010 \pm 0.097 i) \mathrm{meV}$, $t_{3} \approx 0.016 \mathrm{meV}, t_{4} \approx 0.036 \mathrm{meV}$, and $t_{5} \approx 0.119 \mathrm{meV}$. Figure 5 presents the band structure in the effective tightbinding models with (a) $t_{1}$ and $t_{2}$, (b) $t_{1}, t_{2}$, and $t_{5}$, and (c) all the hopping parameters within the distance $r<9 L_{\mathrm{M}}$. The dashed line indicates the original energy band of the effective continuum model.

With hopping terms $t_{1}$ and $t_{2}$ only, the tight-binding model (8) becomes the minimum model introduced in Ref. [4],

$$
\begin{aligned}
H_{0}= & -\mu \sum_{i} \mathbf{c}_{i}^{\dagger} \cdot \mathbf{c}_{i}+\sum_{\langle i j\rangle} t_{1} \mathbf{c}_{i}^{\dagger} \cdot \mathbf{c}_{j}+\text { H.c. } \\
& +\sum_{\langle i j\rangle^{\prime}} \tilde{t}_{2} \mathbf{c}_{i}^{\dagger} \cdot \mathbf{c}_{j}+t_{2}^{\prime}\left(\mathbf{c}_{i}^{\dagger} \times \mathbf{c}_{j}\right)_{z}+\text { H.c. },
\end{aligned}
$$
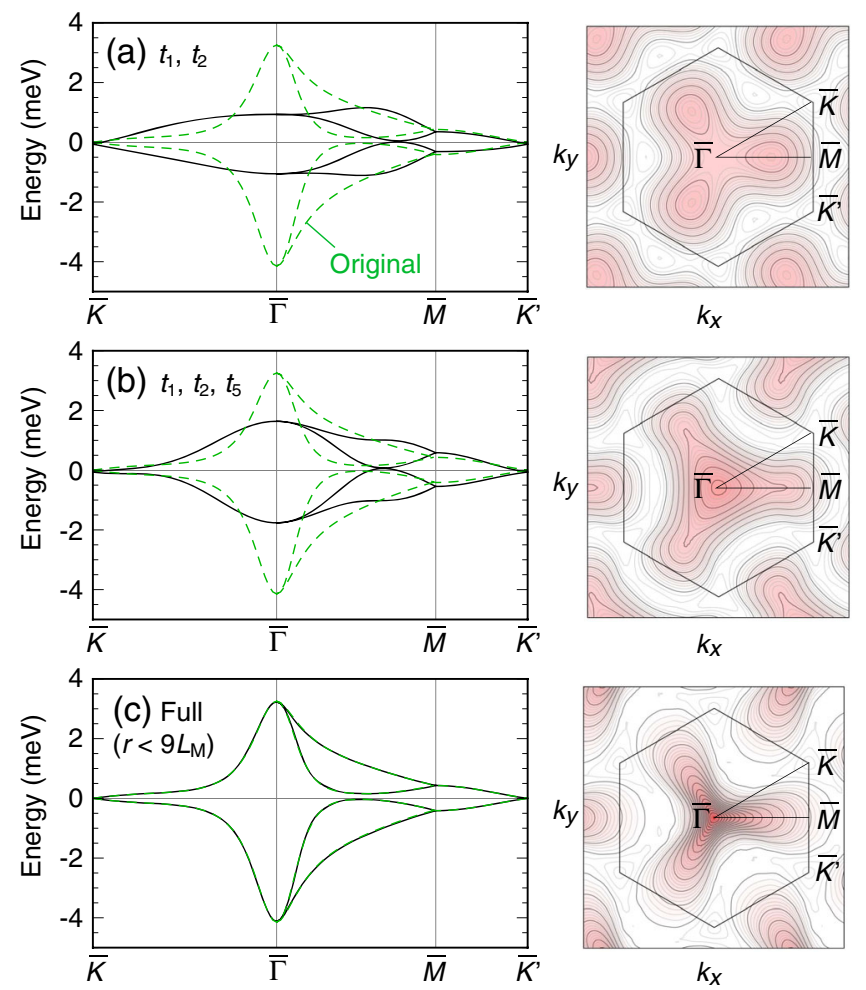

FIG. 5. Band structure in the effective tight-binding model for $\theta=1.05^{\circ}$, with (a) $t_{1}$ and $t_{2}$, (b) $t_{1}, t_{2}$, and $t_{5}$, and (c) all the hopping parameters within the distance $r<9 L_{\mathrm{M}}$. The dashed line indicates the original energy band of the effective continuum model. The right panels show the corresponding contour plots of $E_{1}(\mathbf{k})$ for the valley $\xi=+$.

where $\mathbf{c}_{i}=\left(c_{i, x}, c_{i, y}\right)^{\mathrm{T}}$, with $c_{i, x(y)}$ annihilating an electron with a $p_{x(y)}$ orbital at site $i, c_{j \xi}=\left(c_{j x}+i \xi c_{j y}\right) / \sqrt{2}$. Here, $\mu$ is the on-site chemical potential, $\tilde{t}_{2}=\operatorname{Re}\left(t_{2}\right), t_{2}^{\prime}=$ $\operatorname{Im}\left(t_{2}\right)$, and the sum over $\langle i j\rangle^{\prime}$ includes bonds with length $\sqrt{3} L_{\mathrm{M}}$ along three directions: $\hat{\boldsymbol{x}}, C_{3 z} \hat{\boldsymbol{x}}$, and $C_{3 z}^{2} \hat{\boldsymbol{x}}$. The minimum tight-binding model (9) gives rise to a spectrum with Dirac nodes at $\bar{K}, \bar{K}^{\prime}$ points. Notice that $t_{1}$ denotes hopping between two sublattices, and we can always make $t_{1}$ real by properly choosing the relative phase between sublattices. The $t_{2}^{\prime}$ term describes the hexagonal warping effect in orbital space, which is responsible for band splittings along $\bar{\Gamma} \bar{M}$ lines as shown in Fig. 5.

The symmetry group $G$ allows finite gaps at $\bar{K}, \bar{K}^{\prime}$ points [4]. However, because of the approximate sublattice symmetry at small twist angles [31], we can introduce an additional $\mathbb{Z}_{2}$ symmetry $g: c_{\mathbf{R} \xi} \rightarrow c_{-\mathbf{R},-\xi}$, which combines twofold rotation in real space and a chirality flip in orbital space. In the presence of $g$ and original symmetry group $G$, the gapless Dirac nodes at $\bar{K}, \bar{K}^{\prime}$ points are guaranteed. The minimum model (9) satisfies both $G$ and $g$.

With this additional $\mathbb{Z}_{2}$ symmetry $g$, we then consider additional hopping terms $t_{3}, t_{4}$, and $t_{5}$. As finite $\operatorname{Im}\left(t_{3}\right)$ obeys $G$ while it violates $g$, we find $\operatorname{Im}\left(t_{3}\right)=0$ from our 
numerical calculation of hopping integrals. The nonzero $\tilde{t}_{3} \equiv \operatorname{Re}\left(t_{3}\right), t_{4}, t_{5}$ terms preserve both $G$ and $g$ and quantitatively modify the band structure of the minimum model (9). In fact, including $t_{1}$ to $t_{5}$, we have $m_{e, h}^{-1}=$ $3\left(\tilde{t}_{3}-3 \tilde{t}_{2}\right) \mp\left|\frac{1}{2} t_{1}+2 t_{4}+7 t_{5}\right|$ and $v=(\sqrt{3} / 2)\left|t_{1}-2 t_{4}-t_{5}\right|$, where $m_{e, h}$ denote effective masses at the $\bar{\Gamma}$ point on the electron and hole sides, respectively, and $v$ is the Fermi velocity at the $\bar{K}, \bar{K}^{\prime}$ points.

We can also incorporate the effect of intervalley coupling in the effective tight-binding model by introducing U(1)breaking hopping terms such as those in Ref. [4], which may explain Landau-level-degeneracy lifting in experiments. A detailed analysis of Dirac nodes and mass generation will be presented in a forthcoming work.

\section{ELECTRON-ELECTRON INTERACTION}

We can calculate the electron-electron interaction parameters between the Wannier orbitals directly from the wave functions obtained above. The direct Coulomb interaction $V$ and the exchange interaction $J$ between $|\mathbf{R}, m\rangle$ and $\left|\mathbf{R}^{\prime}, m^{\prime}\right\rangle$ are defined by

$$
V_{\mathbf{R}^{\prime} m^{\prime}, \mathbf{R} m}=\sum_{X X^{\prime}} \iint d \mathbf{r} d \mathbf{r}^{\prime}\left|\psi_{\mathbf{R}^{\prime} m^{\prime}}^{X^{\prime}}\left(\mathbf{r}^{\prime}\right)\right|^{2} \frac{e^{2}}{\epsilon\left|\mathbf{r}-\mathbf{r}^{\prime}\right|}\left|\psi_{\mathbf{R} m}^{X}(\mathbf{r})\right|^{2},
$$

$$
\begin{aligned}
J_{\mathbf{R}^{\prime} m^{\prime}, \mathbf{R} m}= & \sum_{X X^{\prime}} \iint d \mathbf{r} d \mathbf{r}^{\prime} \\
& \times \psi_{\mathbf{R}^{\prime} m^{\prime}}^{X^{\prime} *}\left(\mathbf{r}^{\prime}\right) \psi_{\mathbf{R} m}^{X *}(\mathbf{r}) \frac{e^{2}}{\epsilon\left|\mathbf{r}-\mathbf{r}^{\prime}\right|} \psi_{\mathbf{R}^{\prime} m^{\prime}}^{X}(\mathbf{r}) \psi_{\mathbf{R} m}^{X^{\prime}}\left(\mathbf{r}^{\prime}\right),
\end{aligned}
$$

where $\epsilon$ is the dielectric constant induced by the electrons in other bands and by the external environment (e.g., the substrate). The direct term is the classical Coulomb interaction, and it works for any combination of spin and valley. On the other hand, the exchange interaction works only for the same spin and the same valley. Rigorously speaking, the exchange term between different valleys (and the same spin) is not exactly zero, but there, the integral of $e^{i\left(\mathbf{K}_{+}-\mathbf{K}_{-}\right) \cdot\left(\mathbf{r}-\mathbf{r}^{\prime}\right)} /\left|\mathbf{r}-\mathbf{r}^{\prime}\right|$ in Eq. (11) becomes much smaller than that for the same valley, so we neglect it.

We label the direct interaction terms at different distances as $V_{0}, V_{1}, V_{2} \ldots$ as in Fig. 6(a), where $V_{0}$ is the on-site interaction, $V_{1}$ is the nearest-neighbor interaction, and so forth. Similarly, the exchange terms can be labeled as $J_{1}, J_{2} \ldots$, where $J_{0}$ does not exist because of the Pauli principle. The calculated interaction parameters are listed in Table I. Here, we notice that the on-site interaction $V_{0}$ is not much greater than others, but it has a similar magnitude as the nearest-neighbor interaction $V_{1}$. The further interactions $V_{2}$ and $V_{3}$ are more than half of $V_{0}$. This case
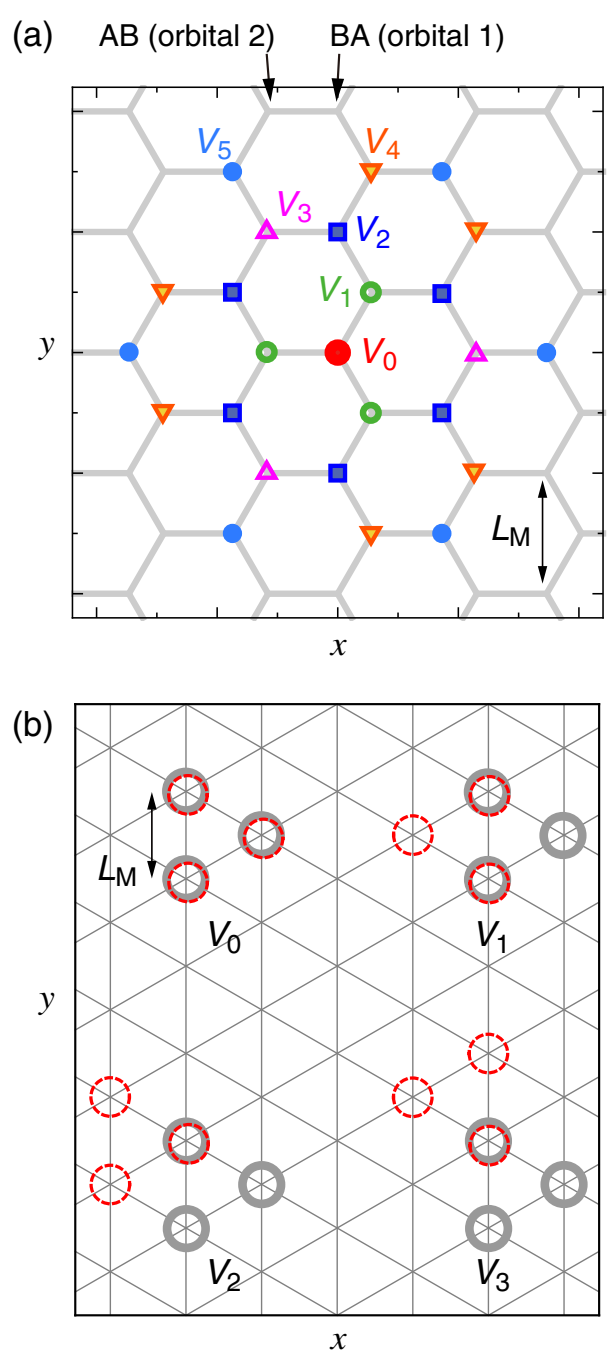

FIG. 6. (a) Labeling of the direct Coulomb interaction at different distances. Note that $V_{0}, V_{1}, V_{2} \ldots$ represent the potential amplitudes between the origin and the indicated lattice points. (b) Overlapping of two Wannier orbitals in the configuration $V_{0}$, $V_{1}, V_{2}, V_{3}$. The three circles of the same line type represent the three peaks of a single Wannier state (Fig. 3).

is quite different from the usual Hubbard-type models where $V_{0}$ dominates the interaction effect. The peculiar distance dependence of the Coulomb interaction in this model is closely related to the three-peak structure of the

TABLE I. Direct interaction $V_{n}$ and the exchange interaction $J_{n}$ for the Wannier orbitals in units of $e^{2} /\left(\epsilon L_{\mathrm{M}}\right)$. The definition of $V_{0}, V_{1} \ldots$ is presented in Fig. 6(a). Here, $V_{n}^{(\text {approx })}$ is the direct interaction term estimated by the point-charge approximation (see the text).

\begin{tabular}{lcccccc}
\hline \hline$n$ & 0 & 1 & 2 & 3 & 4 & 5 \\
\hline$V_{n}$ & 1.857 & 1.533 & 1.145 & 1.068 & 0.697 & 0.614 \\
$V_{n}^{\text {(approx) }}$ & 1.857 & 1.524 & 1.136 & 1.081 & 0.679 & 0.610 \\
$J_{n}$ & $N / \mathrm{A}$ & 0.376 & 0.0645 & 0.010 & 0.014 & 0.001 \\
\hline \hline
\end{tabular}


Wannier orbital. For a single electron, each of the three peaks accommodates the electric charge of $-e / 3$, and thus, the interacting potential between two electrons can be written as a summation over the nine combinations of those fractional charges. The direct Coulomb potential between two fractional charges located at the same peak (i.e., the "on-site interaction" for the fractional charges) is $u_{0} \approx(e / 3)^{2} / \epsilon /\left(0.28 L_{\mathrm{M}}\right)$, while the potential between different peaks is well approximated by that for the point charges, i.e., $(e / 3)^{2} / \epsilon / r$, where $r$ is the distance between the peak centers. The direct interaction terms estimated by this approximation are presented as $V_{n}^{(\text {approx) }}$ in Table I, where the error is found to be $1 \%$ or less. (a)

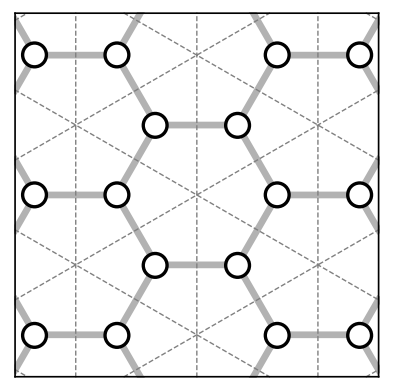

(b)

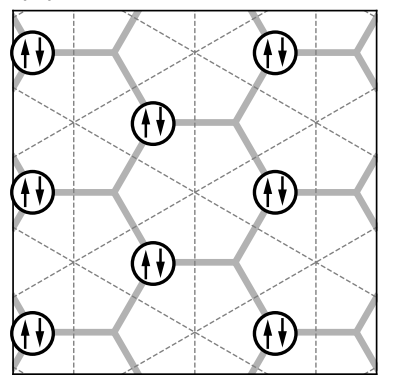

(c)

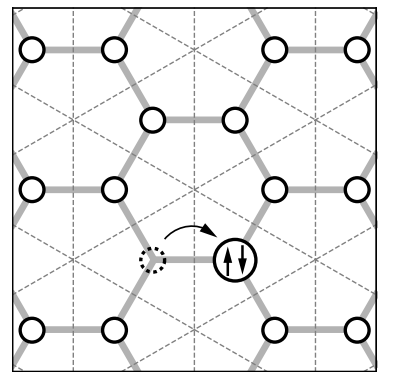

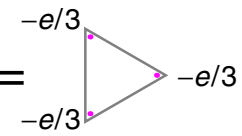
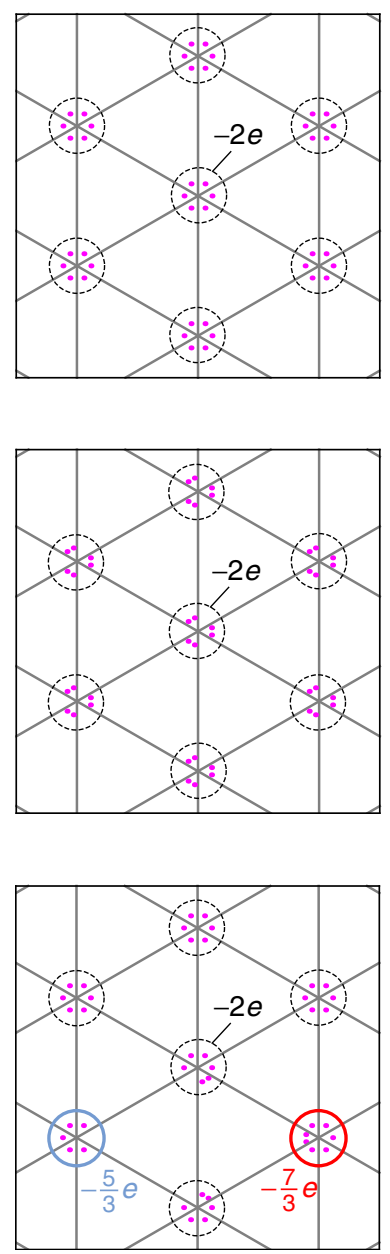

FIG. 7. Several conceivable many-body states illustrated in the honeycomb lattice picture (left panels) and the fractional charge picture (right panels). (a) The homogeneous state where an electron resides at every sublattice. (b) The charge-ordered state where two electrons enter every two sublattices. Opposite arrows in a single site represent two electrons with different spins or valley pseudospins. (c) An excited state from state (a), where an electron is transferred from a single site to another.
Obviously, the dominant contribution to $V_{n}$ comes from the on-site part $u_{0}$. As shown in Fig. 6(b), the electrons located at the same orbital $\left(V_{0}\right)$ share all three peaks, the nearest-neighbor configuration $\left(V_{1}\right)$ the two peaks, and the next-nearest ones $\left(V_{2}, V_{3}\right)$ a single peak. Therefore, the on-site interactions of the fractional charges included in $V_{0}, V_{1}, V_{2}, V_{3}$ are $3 u_{0}, 2 u_{0}, u_{0}, u_{0}$, respectively, and this result explains the dominant part of the relative amplitudes of $V_{n}$ 's. These relatively long-range electron-electron interactions can potentially modify the hopping parameters and hence renormalize the low-energy band structure.

At the filling of two electrons per supercell, in particular, the triangular charge distribution results in an unexpected coincidence in the direct Coulomb energy between two different many-body states shown in Fig. 7, with (a) a homogeneous state where an electron resides at every sublattice of the honeycomb lattice, and (b) a chargeordered state where two electrons enter every two sublattices. It may seem that the direct Coulomb energy in (b) is greater than in (a) because of the double occupancy. However, since an electron at the honeycomb site is actually composed of three $1 / 3$ charges at the triangle corners, states (a) and (b) have nearly identical charge distribution, as shown in the right panels, where the charge of $-2 e$ is registered to every AA spot. Considering that the direct Coulomb interaction is very well approximated by the simple point-charge model as argued above, the total direct energies of (a) and (b) must be nearly equal. The competing nature of the two completely different states may suggest a nontrivial many-body ground state. For further consideration, we need to include the exchange interaction and also the kinetic energy. Lastly, Fig. 7(c) illustrates an excitation from state (a), where an electron is transferred from a single honeycomb site to another. This actually corresponds to a pair creation of the fractional charges $( \pm 1 / 3) e$ as shown in the right panel. This is another intriguing property at this filling factor.

\section{CONCLUSION}

An extended Hubbard model is obtained for the nearly flat band in the low-angle TBG by starting from the Bloch states in a realistic continuum model. The obtained Wannier localized state is centered at the $\mathrm{AB}$ or $\mathrm{BA}$ spot to form a honeycomb lattice. The wave function of the Wannier orbital takes a triangular form, which peaks at three AA spots surrounding the center, and it leads to a competition between the on-site interaction and the neighboring interaction. At the filling of two electrons per supercell, in particular, we have an unusual degeneracy of the chargeordered state and a homogeneous state, which implies a nontrivial nature of the ground state. The detailed studies for the many-body ground states in this model will be left for future works. 


\section{ACKNOWLEDGMENTS}

M. K. thanks Pilkyung Moon and Nguyen N. T. Nam for fruitful discussions. M. K. acknowledges the financial support of JSPS KAKENHI Grant No. JP17K05496. N.F. Q. Y. and L. F. are supported by the DOE Office of Basic Energy Sciences, Division of Materials Sciences and Engineering under Award No. DE-SC0010526. L. F. is partly supported by the David and Lucile Packard Foundation. T. K. is supported by JSPS KAKENHI Grant No. JP18K03442 and JST PRESTO Grant No. JPMJPR15N5. K. K. is supported by JSPS KAKENHI Grant No. JP18H01860.

Note added.-Recently, we became aware of a preprint that reports the maximally localized Wannier states for TBG [46].

\section{APPENDIX: DERIVATION OF THE EFFECTIVE CONTINUUM MODEL UNDER CORRUGATION}

We derive the effective interlayer interaction of a corrugated TBG in Eq. (3), following the method in Ref. [34]. We start from the single-orbital tight-binding model for the $p_{z}$ orbital of carbon atoms. We assume that the transfer integral between any two orbitals is written in terms of the Slater-Koster form as

$$
\begin{aligned}
-t(\mathbf{R}) & =V_{p p \pi}\left[1-\left(\frac{\mathbf{R} \cdot \mathbf{e}_{z}}{R}\right)^{2}\right]+V_{p p \sigma}\left(\frac{\mathbf{R} \cdot \mathbf{e}_{z}}{R}\right)^{2}, \\
V_{p p \pi} & =V_{p p \pi}^{0} e^{-\left(R-a_{0}\right) / r_{0}}, \quad V_{p p \sigma}=V_{p p \sigma}^{0} e^{-\left(R-d_{0}\right) / r_{0}} .
\end{aligned}
$$

Here, $\mathbf{e}_{z}$ is the unit vector perpendicular to the graphene plane, $a_{0}=a / \sqrt{3} \approx 0.142 \mathrm{~nm}$ is the distance of neighboring $A$ and $B$ sites on graphene, and $d_{0} \approx 0.335 \mathrm{~nm}$ is the interlayer spacing of graphite. The parameter $V_{p p \pi}^{0}$ is the transfer integral between the nearest-neighbor atoms on graphene, and $V_{p p \sigma}^{0}$ is the transfer integral between vertically located atoms on the neighboring layers of graphite. We take $V_{p p \pi}^{0} \approx-2.7 \mathrm{eV}, V_{p p \sigma}^{0} \approx 0.48 \mathrm{eV}$ to fit the dispersions of monolayer graphene [34]. Here, $r_{0}$ is the decay length of the transfer integral, and it is chosen as $0.184 a$ so that the next-nearest intralayer coupling becomes $0.1 V_{p p \pi}^{0}$.

To construct the Hamiltonian matrix, we define the Bloch wave bases as

$$
\begin{aligned}
\left|\mathbf{k}, A_{l}\right\rangle & =\frac{1}{\sqrt{N}} \sum_{\mathbf{R}_{A_{l}}} e^{i \mathbf{k} \cdot \mathbf{R}_{A_{l}}}\left|\mathbf{R}_{A_{l}}\right\rangle, \\
\left|\mathbf{k}, B_{l}\right\rangle & =\frac{1}{\sqrt{N}} \sum_{\mathbf{R}_{B_{l}}} e^{i \mathbf{k} \cdot \mathbf{R}_{B_{l}}}\left|\mathbf{R}_{B_{l}}\right\rangle,
\end{aligned}
$$

where the position $\mathbf{R}_{A_{l}}\left(\mathbf{R}_{B_{l}}\right)$ runs over all $A(B)$ sites on the layer $l(=1,2), N$ is the number of the monolayer's unit cell in the whole system, and $\mathbf{k}$ is the two-dimensional Bloch wave vector defined in the first Brillouin zone of the monolayer on layer $l$.

For the interlayer coupling, we first consider a nonrotated bilayer graphene with $\theta=0$ and a constant in-plane displacement $\boldsymbol{\delta}$ from AA stacking. The unit cell is spanned by the monolayer's lattice vectors, $\mathbf{a}_{1}=a(1,0)$ and $\mathbf{a}_{2}=$ $a(1 / 2, \sqrt{3} / 2)$, which are now shared by both layers. Then, the lattice points of the sublattice $X\left(=A_{1}, B_{1}\right.$, $\left.A_{2}, B_{2}\right)$ are given by

$$
\begin{aligned}
& \mathbf{R}_{A_{1}}=n_{1} \mathbf{a}_{1}+n_{2} \mathbf{a}_{2}+\boldsymbol{\tau}_{A_{1}}, \\
& \mathbf{R}_{B_{1}}=n_{1} \mathbf{a}_{1}+n_{2} \mathbf{a}_{2}+\boldsymbol{\tau}_{B_{1}}, \\
& \mathbf{R}_{A_{2}}=n_{1} \mathbf{a}_{1}+n_{2} \mathbf{a}_{2}+\boldsymbol{\tau}_{A_{2}}+\boldsymbol{\delta}+d(\boldsymbol{\delta}) \mathbf{e}_{z}, \\
& \mathbf{R}_{B_{2}}=n_{1} \mathbf{a}_{1}+n_{2} \mathbf{a}_{2}+\boldsymbol{\tau}_{B_{2}}+\boldsymbol{\delta}+d(\boldsymbol{\delta}) \mathbf{e}_{z} .
\end{aligned}
$$

Here, $\boldsymbol{\tau}_{A_{1}}=\boldsymbol{\tau}_{A_{2}}=0, \boldsymbol{\tau}_{B_{1}}=\boldsymbol{\tau}_{B_{2}}=-\boldsymbol{\tau}_{1}$, with $\boldsymbol{\tau}_{1}=\left(2 \mathbf{a}_{2}-\right.$ $\left.\mathbf{a}_{1}\right) / 3=(0, a / \sqrt{3})$, and $d(\boldsymbol{\delta})$ is the optimized interlayer distance, which generally depends on $\boldsymbol{\delta}$. Note that $d(\boldsymbol{\delta})$ is a periodic function of $\boldsymbol{\delta}$ with periods of $\mathbf{a}_{1}$ and $\mathbf{a}_{2}$ because the interlayer shift by a lattice vector just gives the equivalent structure. It is known that the interlayer spacing takes the maximum value $d_{\mathrm{AA}}$ at AA stacking $(\boldsymbol{\delta}=0)$ and the minimum at $d_{\mathrm{AB}}$ at $\mathrm{AB}$ stacking $\left(\boldsymbol{\delta}=\boldsymbol{\tau}_{1}\right)$ [47]. Here, we adopt $d_{\mathrm{AA}}=0.360 \mathrm{~nm}$ and $d_{\mathrm{AB}}=0.335 \mathrm{~nm}[41,47]$. The distance at intermediate $\delta$ can be interpolated by

$$
d(\boldsymbol{\delta})=d_{0}+2 d_{1} \sum_{j=1}^{3} \cos \mathbf{a}_{i}^{*} \boldsymbol{\delta},
$$

where $\mathbf{a}_{3}^{*}=-\mathbf{a}_{1}^{*}-\mathbf{a}_{2}^{*}$, and

$$
\begin{gathered}
d_{0}=\frac{1}{3}\left(d_{\mathrm{AA}}+2 d_{\mathrm{AB}}\right), \\
d_{1}=\frac{1}{9}\left(d_{\mathrm{AA}}-d_{\mathrm{AB}}\right) .
\end{gathered}
$$

The interlayer matrix element from $X=A_{1}, B_{1}$ to $X^{\prime}=A_{2}, \quad B_{2}$ is obtained by taking all the transfer integrals between atoms of layer 1 and layer 2. It is explicitly written as

$$
\begin{aligned}
U_{X^{\prime} X}(\mathbf{k}, \boldsymbol{\delta}) \equiv & \left\langle\mathbf{k}, X^{\prime}|H| \mathbf{k}, X\right\rangle \\
= & \sum_{n_{1}, n_{2}}-t\left[n_{1} \mathbf{a}_{1}+n_{2} \mathbf{a}_{2}+\boldsymbol{\tau}_{X^{\prime} X}+\boldsymbol{\delta}+d(\boldsymbol{\delta}) \mathbf{e}_{z}\right] \\
& \times \exp \left[-i \mathbf{k} \cdot\left(n_{1} \mathbf{a}_{1}+n_{2} \mathbf{a}_{2}+\boldsymbol{\tau}_{X^{\prime} X}+\boldsymbol{\delta}\right)\right],
\end{aligned}
$$

where $\boldsymbol{\tau}_{X^{\prime} X}=\boldsymbol{\tau}_{X^{\prime}}-\boldsymbol{\tau}_{X}$. Note that $U_{X^{\prime} X}(\mathbf{k}, \boldsymbol{\delta})$ is also a periodic function of $\boldsymbol{\delta}$ with periods $\mathbf{a}_{1}$ and $\mathbf{a}_{2}$, and therefore, it can be written as a Fourier expansion, 


$$
\begin{aligned}
U_{X^{\prime} X}(\mathbf{k}, \boldsymbol{\delta}) \equiv & \left\langle\mathbf{k}, X^{\prime}|H| \mathbf{k}, X\right\rangle \\
= & \sum_{m_{1}, m_{2}} \tilde{U}_{X^{\prime} X}\left(m_{1} \mathbf{a}_{1}^{*}+m_{2} \mathbf{a}_{2}^{*}+\mathbf{k}\right) \\
& \times \exp \left[i\left(m_{1} \mathbf{a}_{1}^{*}+m_{2} \mathbf{a}_{2}^{*}\right) \cdot\left(\boldsymbol{\delta}+\boldsymbol{\tau}_{X^{\prime} X}\right)\right] .
\end{aligned}
$$

Here, we define

$$
\tilde{U}_{X^{\prime} X}(\mathbf{q})=-\frac{1}{S_{0}} \int t\left[\mathbf{R}+d\left(\mathbf{R}-\boldsymbol{\tau}_{X^{\prime} X}\right) \mathbf{e}_{z}\right] e^{-i \mathbf{q} \cdot \mathbf{R}} d^{2} \mathbf{R},
$$

where $S_{0}=(\sqrt{3} / 2) a^{2}$ is the unit area of monolayer graphene, and the integral in $\mathbf{R}$ is over the infinite twodimensional space. Note that $\tilde{U}_{X^{\prime} X}(\mathbf{q})$ is circular symmetric and only depends on $|\mathbf{q}|$. Since $t(\mathbf{R})$ exponentially decays in $R \sim r_{0}$, the Fourier transform $\tilde{U}_{X^{\prime} X}(\mathbf{q})$ decays in $q \sim 1 / r_{0}$.

When we rotate one graphene layer to another by a small twist angle $\theta$, the local lattice structure in the moiré pattern is approximately viewed as a nonrotated bilayer graphene, where the displacement $\boldsymbol{\delta}$ slowly depends on the position $\mathbf{r}$ in accordance with [38]

$$
\boldsymbol{\delta}(\mathbf{r})=[R(\theta / 2)-R(-\theta / 2)] \mathbf{r} .
$$

The interlayer matrix element for valley $\xi$ is then approximately written as $U_{X^{\prime} X}\left[\mathbf{K}_{\xi}, \boldsymbol{\delta}(\mathbf{r})\right]$ [34]. Using Eqs. (A8) and (A10), we obtain

$$
\begin{aligned}
U_{X^{\prime} X} & {\left[K_{\xi}, \boldsymbol{\delta}(\mathbf{r})\right] } \\
= & \sum_{m_{1}, m_{2}} \tilde{U}_{X^{\prime} X}\left(m_{1} \mathbf{a}_{1}^{*}+m_{2} \mathbf{a}_{2}^{*}+\mathbf{K}_{\xi}\right) \\
& \quad \times \exp \left[i\left(m_{1} \mathbf{a}_{1}^{*}+m_{2} \mathbf{a}_{2}^{*}\right) \cdot \boldsymbol{\tau}_{X^{\prime} X}\right] \\
& \quad \times \exp \left[i\left(m_{1} \mathbf{G}_{1}^{\mathrm{M}}+m_{2} \mathbf{G}_{2}^{\mathrm{M}}\right) \cdot \mathbf{r}\right],
\end{aligned}
$$

where we use the relationship $\mathbf{a}_{i}^{*} \cdot \boldsymbol{\delta}(\mathbf{r})=\mathbf{G}_{i}^{\mathrm{M}} \cdot \mathbf{r}$. Now, we see that Eq. (A11) is periodic in $\mathbf{r}$ with the moiré reciprocal vectors $\mathbf{G}_{i}^{\mathrm{M}}$. Since $\tilde{U}_{X^{\prime} X}(\mathbf{q})$ rapidly decays in $q$, we only need a few Fourier components in Eq. (A11). By taking the largest three terms given by $\left(m_{1}, m_{2}\right)=(0,0), \xi(1,0)$, $\xi(1,1)$, we have the Hamiltonian in Eq. (3), where

$$
\begin{aligned}
u & =-\frac{1}{S_{0}} \int t\left[\mathbf{R}+d(\mathbf{R}) \mathbf{e}_{z}\right] e^{-i \mathbf{K}_{\xi} \cdot \mathbf{R}} d^{2} \mathbf{R}, \\
u^{\prime} & =-\frac{1}{S_{0}} \int t\left[\mathbf{R}+d\left(\mathbf{R}-\boldsymbol{\tau}_{1}\right) \mathbf{e}_{z}\right] e^{-i \mathbf{K}_{\xi} \cdot \mathbf{R}} d^{2} \mathbf{R} .
\end{aligned}
$$

We obtain $u=0.0797 \mathrm{eV}$ and $u^{\prime}=0.0975 \mathrm{eV}$ for the present parameters. In a flat TBG, the interlayer distance $d(\boldsymbol{\delta})$ is constant, and therefore, we have $u=u^{\prime}$ [34].

[1] Y. Cao, V. Fatemi, S. Fang, K. Watanabe, T. Taniguchi, E. Kaxiras, and P. Jarillo-Herrero, Unconventional Superconductivity in Magic-Angle Graphene Superlattices, Nature (London) 556, 43 (2018).
[2] Y. Cao, V. Fatemi, A. Demir, S. Fang, S. L. Tomarken, J. Y. Luo, J. D. Sanchez-Yamagishi, K. Watanabe, T. Taniguchi, E. Kaxiras, R. C. Ashoori, and P. Jarillo-Herrero, Correlated Insulator Behaviour at Half-Filling in Magic-Angle Graphene Superlattices, Nature (London) 556, 80 (2018).

[3] C. Xu and L. Balents, Topological Superconductivity in Twisted Multilayer Graphene, arXiv:1803.08057.

[4] N. F. Q. Yuan and L. Fu, A Model for Metal-Insulator Transition in Graphene Superlattices and Beyond, Phys. Rev. B 98, 045103 (2018).

[5] H. C. Po, L. Zou, A. Vishwanath, and T. Senthil, Origin of Mott Insulating Behavior and Superconductivity in Twisted Bilayer Graphene, arXiv:1803.09742.

[6] B. Roy and V. Juricic, Unconventional Superconductivity in Nearly Flat Bands in Twisted Bilayer Graphene, arXiv: 1803.11190.

[7] H. Guo, X. Zhu, S. Feng, and R. T. Scalettar, Pairing Symmetry of Interacting Fermions on Twisted Bilayer Graphene Superlattice, Phys. Rev. B 97, 235453 (2018).

[8] B. Padhi, C. Setty, and P. W. Phillips, Wigner Crystallization in Lieu of Mottness in Twisted Bilayer Graphene, arXiv: 1804.01101.

[9] J. F. Dodaro, S. A. Kivelson, Y. Schattner, X.-Q. Sun, and C. Wang, Phases of a Phenomenological Model of Twisted Bilayer Graphene, arXiv:1804.03162.

[10] T. Huang, L. Zhang, and T. Ma, Antiferromagnetically Ordered Mott Insulator and $d+i d$ Superconductivity in Twisted Bilayer Graphene: A Quantum Monte Carlo Study, arXiv:1804.06096.

[11] L. Zhang, Low-Energy Moiré Band Formed by Dirac Zero Modes in Twisted Bilayer Graphene, arXiv:1804.09047.

[12] S. Ray and T. Das, Wannier Pairs in the Superconducting Twisted Bilayer Graphene and Related Systems, arXiv: 1804.09674.

[13] C.-C. Liu, L.-D. Zhang, W.-Q. Chen, and F. Yang, Chiral SDW and $d+i d$ Superconductivity in the Magic-Angle Twisted Bilayer-Graphene, arXiv:1804.10009.

[14] X. Y. Xu, K. T. Law, and P. A. Lee, Kekulé Valence Bond Order in an Extended Hubbard Model on the Honeycomb Lattice, with Possible Applications to Twisted Bilayer Graphene, arXiv:1805.00478.

[15] T. J. Peltonen, R. Ojajärvi, and T. T. Heikkilä, Mean-Field Theory for Superconductivity in Twisted Bilayer Graphene, arXiv:1805.01039.

[16] M. Fidrysiak, M. Zegrodnik, and J. Spałek, Unconventional Topological Superconductivity and Phase Diagram for a Two-Orbital Model of Twisted Bilayer Graphene, arXiv: 1805.01179.

[17] C. Berger, Z. Song, X. Li, X. Wu, N. Brown, C. Naud, D. Mayou, T. Li, J. Hass, A. N. Marchenkov, E. H. Conrad, P. N. First, and W. A. de Heer, Electronic Confinement and Coherence in Patterned Epitaxial Graphene, Science 312, 1191 (2006).

[18] J. Hass, R. Feng, J. E. Millan-Otoya, X. Li, M. Sprinkle, P. N. First, W. A. de Heer, E. H. Conrad, and C. Berger, Structural Properties of the Multilayer Graphene/4H-SiC (0001) System as Determined by Surface X-Ray Diffraction, Phys. Rev. B 75, 214109 (2007).

[19] J. Hass, F. Varchon, J. E. Millan-Otoya, M. Sprinkle, N. Sharma, W. A. de Heer, C. Berger, P. N. First, L. Magaud, 
and E. H. Conrad, Why Multilayer Graphene on 4H-SiC (0001) Behaves Like a Single Sheet of Graphene, Phys. Rev. Lett. 100, 125504 (2008).

[20] G. Li, A. Luican, J. M. B. Lopes dos Santos, A. H. C. Neto, A. Reina, J. Kong, and E. Y. Andrei, Observation of van Hove Singularities in Twisted Graphene Layers, Nat. Phys. 6, 109 (2010).

[21] D. L. Miller, K. D. Kubista, G. M. Rutter, M. Ruan, W. A. de Heer, P. N. First, and J. A. Stroscio, Structural Analysis of Multilayer Graphene via Atomic Moiré Interferometry, Phys. Rev. B 81, 125427 (2010).

[22] A. Luican, G. Li, A. Reina, J. Kong, R. R. Nair, K. S. Novoselov, A. K. Geim, and E. Y. Andrei, Single-Layer Behavior and Its Breakdown in Twisted Graphene Layers, Phys. Rev. Lett. 106, 126802 (2011).

[23] J. M. B. Lopes dos Santos, N. M. R. Peres, and A. H. Castro Neto, Graphene Bilayer with a Twist: Electronic Structure, Phys. Rev. Lett. 99, 256802 (2007).

[24] E. J. Mele, Commensuration and Interlayer Coherence in Twisted Bilayer Graphene, Phys. Rev. B 81, 161405 (2010).

[25] G. Trambly de Laissardière, D. Mayou, and L. Magaud, Localization of Dirac Electrons in Rotated Graphene Bilayers, Nano Lett. 10, 804 (2010).

[26] S. Shallcross, S. Sharma, E. Kandelaki, and O. A. Pankratov, Electronic Structure of Turbostratic Graphene, Phys. Rev. B 81, 165105 (2010).

[27] E. S. Morell, J. D. Correa, P. Vargas, M. Pacheco, and Z. Barticevic, Flat Bands in Slightly Twisted Bilayer Graphene: Tight-Binding Calculations, Phys. Rev. B 82, 121407 (2010).

[28] R. Bistritzer and A. H. MacDonald, Moiré Bands in Twisted Double-Layer Graphene, Proc. Natl. Acad. Sci. U.S.A. 108, 12233 (2011).

[29] M. Kindermann and P. N. First, Local Sublattice-Symmetry Breaking in Rotationally Faulted Multilayer Graphene, Phys. Rev. B 83, 045425 (2011).

[30] L. Xian, S. Barraza-Lopez, and M. Y. Chou, Effects of Electrostatic Fields and Charge Doping on the Linear Bands in Twisted Graphene Bilayers, Phys. Rev. B 84, 075425 (2011).

[31] J. M. B. Lopes dos Santos, N. M. R. Peres, and A. H. Castro Neto, Continuum Model of the Twisted Graphene Bilayer, Phys. Rev. B 86, 155449 (2012).

[32] P. Moon and M. Koshino, Energy Spectrum and Quantum Hall Effect in Twisted Bilayer Graphene, Phys. Rev. B 85, 195458 (2012).

[33] G. Trambly de Laissardiere, D. Mayou, and L. Magaud, Numerical Studies of Confined States in Rotated Bilayers of Graphene, Phys. Rev. B 86, 125413 (2012).

[34] P. Moon and M. Koshino, Optical Absorption in Twisted Bilayer Graphene, Phys. Rev. B 87, 205404 (2013).
[35] M. Koshino, Interlayer Interaction in General Incommensurate Atomic Layers, New J. Phys. 17, 015014 (2015).

[36] M. Koshino and P. Moon, Electronic Properties of Incommensurate Atomic Layers, J. Phys. Soc. Jpn. 84, 121001 (2015).

[37] Y. Cao, J. Y. Luo, V. Fatemi, S. Fang, J. D. SanchezYamagishi, K. Watanabe, T. Taniguchi, E. Kaxiras, and P. Jarillo-Herrero, Superlattice-Induced Insulating States and Valley-Protected Orbits in Twisted Bilayer Graphene, Phys. Rev. Lett. 117, 116804 (2016).

[38] N. N. T. Nam and M. Koshino, Lattice Relaxation and Energy Band Modulation in Twisted Bilayer Graphene, Phys. Rev. B 96, 075311 (2017).

[39] N. Marzari and D. Vanderbilt, Maximally Localized Generalized Wannier Functions for Composite Energy Bands, Phys. Rev. B 56, 12847 (1997).

[40] See Supplemental Material http://link.aps.org/supplemental/ 10.1103/PhysRevX.8.031087 for the symmetry consideration of the Wannier orbitals in the $D_{6}$ structure and other superlattice structures.

[41] K. Uchida, S. Furuya, J.-I. Iwata, and A. Oshiyama, Atomic Corrugation and Electron Localization Due to Moiré Patterns in Twisted Bilayer Graphenes, Phys. Rev. B 90, 155451 (2014).

[42] M. M. van Wijk, A. Schuring, M. I. Katsnelson, and A. Fasolino, Relaxation of Moiré Patterns for Slightly Misaligned Identical Lattices: Graphene on Graphite, 2D Mater. 2, 034010 (2015).

[43] S. Dai, Y. Xiang, and D. J. Srolovitz, Twisted Bilayer Graphene: Moiré with a Twist, Nano Lett. 16, 5923 (2016).

[44] S. K. Jain, V. Juričić, and G. T. Barkema, Structure of Twisted and Buckled Bilayer Graphene, 2D Mater. 4, 015018 (2016).

[45] See Supplemental Material http://link.aps.org/supplemental/ 10.1103/PhysRevX.8.031087. In the data file, each line contains the components of the vector $\mathbf{R}$ in terms of the lattice vectors $\mathbf{L}_{i}^{\mathrm{M}}(i=1,2)$, the orbital indices $m$ and $n$, and the real and imaginary parts of the Hamiltonian matrix element $\langle\mathbf{R}, m|H| \mathbf{0}, n\rangle$. Here, the lattice vectors are taken as $\mathbf{L}_{1}^{\mathrm{M}}=(\sqrt{3} / 2,1 / 2) L_{\mathrm{M}}, \mathbf{L}_{2}^{\mathrm{M}}=(0,1) L_{\mathrm{M}}$, and the sublattice positions of orbitals 1 and 2 in the same unit cell are defined by $\mathbf{r}_{\mathrm{BA}}=(1 / 2, \sqrt{3} / 2)\left(L_{\mathrm{M}} / \sqrt{3}\right)$ and $\mathbf{r}_{\mathrm{AB}}=(-1 / 2$, $\sqrt{3} / 2)\left(L_{\mathrm{M}} / \sqrt{3}\right)$, respectively. We omit the hopping parameters of distance greater than $9 L_{\mathrm{M}}$.

[46] J. Kang and O. Vafek, Symmetry, Maximally Localized Wannier States, and Low Energy Model for the Twisted Bilayer Graphene Narrow Bands, following article, Phys. Rev. X 8, 031088 (2018).

[47] J.-K. Lee, S.-C. Lee, J.-P. Ahn, S.-C. Kim, J. I. B. Wilson, and P. John, The Growth of AA Graphite on (111) Diamond, J. Chem. Phys. 129, 234709 (2008). 